\title{
Constraints on anharmonic corrections of fuzzy dark matter
}

\author{
J.A.R. Cembranos, A.L. Maroto, S.J. Núñez Jareño and H. Villarrubia-Rojo \\ Departamento de Física Teórica, Universidad Complutense de Madrid, \\ E-28040 Madrid, Spain \\ E-mail: cembra@ucm.es, maroto@ucm.es, sjnjareno@fis.ucm.es, \\ hectorvi@ucm.es
}

ABSTRACT: The cold dark matter (CDM) scenario has proved successful in cosmology. However, we lack a fundamental understanding of its microscopic nature. Moreover, the apparent disagreement between CDM predictions and subgalactic-structure observations has prompted the debate about its behaviour at small scales. These problems could be alleviated if the dark matter is composed of ultralight fields $m \sim 10^{-22} \mathrm{eV}$, usually known as fuzzy dark matter (FDM). Some specific models, with axion-like potentials, have been thoroughly studied and are collectively referred to as ultralight axions (ULAs) or axion-like particles (ALPs). In this work we consider anharmonic corrections to the mass term coming from a repulsive quartic self-interaction. Whenever this anharmonic term dominates, the field behaves as radiation instead of cold matter, modifying the time of matter-radiation equality. Additionally, even for high masses, i.e. masses that reproduce the cold matter behaviour, the presence of anharmonic terms introduce a cut-off in the matter power spectrum through its contribution to the sound speed. We analyze the model and derive constraints using a modified version of CLASS and comparing with CMB and large-scale structure data.

Keywords: Cosmology of Theories beyond the SM, Beyond Standard Model

ARXIV EPRINT: 1805.08112 


\section{Contents}

1 Introduction 1

2 Exact evolution 3

3 Averaged evolution $\quad 5$

4 Heuristic constraints on the non-harmonic contribution 8

5 Numerical evolution and constraints $\quad 10$

5.1 Physical effects 11

$\begin{array}{ll}5.2 \text { Observational constraints } & 15\end{array}$

6 Conclusions 16

\section{Introduction}

The evidence collected over the last decades suggests that most of the matter in the universe exists in the form of dark matter (DM), whose effects have only been detected through its gravitational interaction. In particular, the assumption that dark matter is composed of non-relativistic particles, the so-called cold dark matter (CDM), has produced a remarkable concordance with the observational data over a wide range of scales and evolution epochs. It is one of the foundations of the succesful standard cosmological model $\Lambda \mathrm{CDM}$.

Notwithstanding agreement with observations, several ingredients are lacking in our understanding of DM. In the first place, we have been unable to detect any nongravitational interaction of DM. Most of the work in the field is currently devoted to direct, indirect detection and production searches. Owing to this effort it has been possible to tighten the parameter space of the most popular models. This lack of additional interactions makes it more difficult to discriminate between different models. There are many candidates that behave like $\mathrm{CDM}$ on cosmological scales, with masses ranging from the meV of the QCD axion [1] to the $\mathrm{TeV}[2,3]$ and going up to the $100 M_{\odot}$ of the primordial black holes [4]. The other ingredient missing is a precise understanding of the DM behaviour on small, i.e. galactic, scales. Even though most DM models mimic CDM on cosmological scales, their predictions usually differ on smaller scales [5] so they could be discriminated based only on their gravitational effects. In fact, there exist three long-standing debates, questioning the agreement between observations and the CDM theoretical predictions [6, 7], the so-called 'too big to fail' [8], 'missing satellites' [9] and especially the 'core-cusp' problem [10]. The 'core-cusp' problem refers to the discrepancy between the density profiles of CDM halos obtained in $N$-body simulations, that tend to be cuspy in the center, and the ones inferred from observations, that point to the existence of a central 
core. Although these problems are sometimes attributed to baryonic effects unaccounted for in the simulations [11], they remain one of the main challenges of the CDM model.

An interesting alternative that neatly solves the 'core-cusp' problem is fuzzy dark matter (FDM) [12]. In this picture, dark matter is composed of ultralight particles with $m \sim 10^{-22} \mathrm{eV}$, so that its Compton wavelength $\left(\mathrm{m}^{-1}\right)$ reaches astrophysical scales. Then, the formation of cusps is prevented [13]. The wave nature of the particles on the smallest scales makes them impossible to localize. While solving this problem, FDM behaves as a rapidly oscillating coherent scalar field, thus recovering the CDM behaviour on cosmological scales. In his groundbreaking work [14], Turner analyzed a homogeneous oscillating scalar field in an expanding universe. He showed that a rapidly oscillating scalar field with a power-law potential $V(\phi) \propto \phi^{n}$ behaves as a perfect fluid with an effective equation of state $w=(n-2) /(n+2)$. More general expressions can be obtained from a version of the virial theorem [15]. The results of Turner show that a massive scalar field, i.e. harmonic potential, oscillating coherently with a frequency much higher than the expansion rate behaves as CDM, at least at the background level. Afterwards, ultralight scalar fields have been thoroughly studied at the perturbation level [15-19], proving that the same conclusion holds. Perturbations of coherent oscillating scalar fields admit an effective fluid description with an effective sound speed nearly zero, like CDM. The main cosmological signature of these models is the supression of growth at small scales. Below some Jeans scale $k_{J}^{-1}$ the modes do not grow appreciably, translating into a cut-off in the matter power spectrum [18]. Additionally, there are important effects in CMB temperature, lensing and polarization spectra. These effects were analyzed in $[20,21]$ using the publicly available code AxiOnCAMB. Although the work on ultralight fields has been mainly concerned with scalar fields, there are recent results on higher spin fields. It has been shown that abelian vectors at the background [22] and perturbation level [23], non-abelian vectors [24] and arbitrary-spin fields [25] behave in a similar way. Interestingly, the results of [25] show that it is possible to achieve an isotropic model of higher-spin dark matter as long as it is rapidly oscillating.

These ideas have been applied to the axion, a particularly well-motivated DM candidate. The standard QCD axion was initially proposed to solve the strong CP problem [2628 ] in particle physics. Likewise, the appereance of many light scalar fields seems to be a generic feature of different string-theory scenarios. Some of these fields have a similar origin as the QCD axion, arising from the breaking of an approximate shift symmetry, and are usually known as axion-like particles (ALPs) or ultralight axions (ULAs) [29, 30]. ALPs present similar periodic potentials but with a mass much smaller than the QCD axion that could lie in the range of ultralight fields $m \sim 10^{-22} \mathrm{eV}$. While behaving like FDM, ALPs have a rich phenomenology based on their assumed interaction with matter. Aside from the standard searches for axions, there is a wealth of dedicated searches and projected experiments on the lookout for ultralight axions. These include studies of the neutral hydrogen distribution in the universe [31,32], laboratory constraints based on nuclear interactions [33], variation of fundamental constants [34, 35], astrophysical bounds [36-38], gravitational wave searches $[39,40]$ and analysis of CMB spectral distortions [41, 42]. A prominent feature of the model is the presence of anharmonic corrections over the mass 
term in FDM. These corrections arise, to first order, as quartic corrections in the potential with the opposite sign of the mass term, i.e. attractive self-interactions. These effects have been studied, as well as the effect of the full axion potential $[43,44]$ and their effect on the linear matter power spectrum seems to be negligible. However, self-interactions could modify non-linear structures in a significant way [45].

Another possibility involves introducing a positive quartic correction, i.e. repulsive self-interactions. It is more difficult to find particle-physics models in this case [46], but the model is nonetheless well motivated as the simplest modification leading to a stable potential. This modification has been previously analyzed in some works [47-52]. The additional source of pressure from the repulsive self-interactions helps to solve the 'corecusp' problem with larger masses [46]. Additionaly, unlike the axion case, it could explain the formation of vortices in galaxies [53].

In this work we will consider a fuzzy dark matter model with an additional quartic self-interaction. Using a modified version of the cosmological Boltzmann code CLASS [54] and parameter-extraction code MontePython [55] we will constrain the parameters of the model with CMB [56] and large-scale structure (LSS) [57] data. Section 2 presents the model and the relevant equations for background and perturbation evolution. In section 3 , we review the averaging procedure when the field is rapidly oscillating and the effective fluid equations in this case. Section 4 discusses a simplified model and estimates analytic bounds on the parameters, highlighting the main physical effects and the origin of the constraints on the model. In section 5 we present the result of the full numerical analysis and the final constraints on the model, as well as a discussion of its physical origin. Section 6 summarizes the conclusions and prospects for future work.

\section{Exact evolution}

Let us assume a scalar field with Lagrangian

$$
\mathcal{L}=\frac{1}{2} g^{\mu \nu} \partial_{\mu} \phi \partial_{\nu} \phi-V(\phi)
$$

and potential

$$
V(\phi)=\frac{1}{2} m^{2} \phi^{2}+\frac{1}{4} \lambda \phi^{4},
$$

in a homogeneous and isotropic universe with a flat Robertson-Walker metric in conformal time $\eta$

$$
\mathrm{d} s^{2}=a^{2}(\eta)\left(\mathrm{d} \eta^{2}-\mathrm{d} \boldsymbol{x}^{2}\right)
$$

The equation of motion for a homogeneous scalar field in this background is

$$
\ddot{\phi}+2 \mathcal{H} \dot{\phi}+a^{2} V^{\prime}(\phi)=0,
$$

where $\mathcal{H}=\dot{a} / a$ and $\equiv \partial / \partial \eta$. The density and pressure are

$$
\begin{gathered}
\rho=\frac{\dot{\phi}^{2}}{2 a^{2}}+V(\phi), \\
P=\frac{\dot{\phi}^{2}}{2 a^{2}}-V(\phi) .
\end{gathered}
$$


We choose initial conditions

$$
\begin{aligned}
\phi & =\phi_{0}, \\
\dot{\phi} & =0,
\end{aligned}
$$

The value $\phi_{0}$ will be chosen to match the desired energy density $\rho_{\phi}$ today. Section 5 contains more details about how this matching is performed. These are the usual initial conditions when the axion-like particles are produced through a misalignment mechanism [58] and the field starts its evolution frozen. It is important to note that the choice of initial conditions has a deep impact in the subsequent evolution. In [49], the authors considered a case similar to ours, but with an initial velocity $\dot{\phi} \neq 0$. In this case, there is an initial phase of stiff-matter domination, absent in our case, constrained to be short enough not to spoil BBN.

We now introduce scalar perturbations over a flat Robertson-Walker metric. Following the notation of [59], the general form of the perturbations is

$$
\mathrm{d} s^{2}=a^{2}(\eta)\left[(1+2 \Phi) \mathrm{d} \eta^{2}-2 \partial_{i} B \mathrm{~d} x^{i} \mathrm{~d} \eta-\left((1-2 \Psi) \delta_{i j}+2 \partial_{i} \partial_{j} E\right) \mathrm{d} x^{i} \mathrm{~d} x^{j}\right] .
$$

The equation of motion for the scalar field perturbation is

$$
\ddot{\delta \phi}+2 \mathcal{H} \dot{\delta \phi}+\left(k^{2}+a^{2} V^{\prime \prime}\right) \delta \phi=\left(k^{2}(B-\dot{E})+\dot{\Phi}+3 \dot{\Psi}\right) \dot{\phi}-2 a^{2} \Phi V^{\prime},
$$

where the gauge has not yet been fixed. We can introduce a different parameterization, reminiscent of a perfect fluid. The components of the perturbed energy-momentum tensor are [59]

$$
\begin{aligned}
\delta T_{0}^{0} & \equiv \delta \rho_{\phi}=a^{-2}\left(\dot{\phi} \dot{\delta \phi}-\dot{\phi}^{2} \Phi\right)+V^{\prime} \delta \phi, \\
\delta T_{j}^{i} & \equiv-\delta P_{\phi} \delta_{j}^{i}=-\left(a^{-2}\left(\dot{\phi} \dot{\delta \phi}-\dot{\phi}^{2} \Phi\right)-V^{\prime} \delta \phi\right) \delta_{j}^{i}, \\
\delta T_{i}^{0} & \equiv\left(\rho_{\phi}+P_{\phi}\right)\left(v_{\phi}^{i}-\partial_{i} B\right)=a^{-2} \dot{\phi} \partial_{i} \delta \phi .
\end{aligned}
$$

We can rewrite (2.10) in terms of the fluid variables, introducing $\delta=\delta \rho / \rho$ and $u=$ $(1+w)(v-B)$, where $w$ is the equation of state for the scalar field

$$
w \equiv \frac{P}{\rho}=\frac{\dot{\phi}^{2}-2 a^{2} V(\phi)}{\dot{\phi}^{2}+2 a^{2} V(\phi)} .
$$

In the synchronous gauge, the metric variables read

$$
\begin{aligned}
& \Psi=-\frac{1}{6}\left(h-\nabla^{2} \mu\right), \\
& E=\frac{1}{2} \mu, \\
& \Phi=B=0,
\end{aligned}
$$

and the equations of motion are

$$
\begin{aligned}
\dot{\delta} & =-3 \mathcal{H}(1-w) \delta-k u-9 \mathcal{H}^{2}\left(1-c_{\text {ad }}^{2}\right) \frac{u}{k}-\frac{1}{2}(1+w) \dot{h}, \\
\dot{u} & =2 \mathcal{H} u+k \delta+3\left(w-c_{\text {ad }}^{2}\right) \mathcal{H} u,
\end{aligned}
$$


where $c_{\text {ad }}^{2}$ is the adiabatic sound speed

$$
c_{\text {ad }}^{2}=\frac{\dot{P}}{\dot{\rho}}=1+\frac{2}{3} \frac{a^{2} V^{\prime}}{\mathcal{H} \dot{\phi}} .
$$

Following the analysis of [18] we provide the system with initial conditions

$$
\begin{aligned}
& \delta=0, \\
& u=0,
\end{aligned}
$$

valid up to corrections of order $(k \eta)^{4}$. The scalar field starts its evolution frozen in a value $\phi_{0}$ with an equation of state $w \simeq-1$. As the universe expands the field starts rolling down the potential, when it reaches the minimum it undergoes rapid oscillations. These oscillations occur when the effective frequency $\omega_{\text {eff }} \sim \sqrt{V^{\prime \prime}(\phi)}$ is bigger than the friction term $\mathcal{H}$, so once the scalar field starts oscillating its frequency becomes much larger than the expansion rate, the inverse of the evolution time scale of the background.

On the numerical side, this means that it becomes prohibitely expensive to compute the exact evolution of the field, following every oscillation. However, the huge difference between time scales allows us to average the equations of motion and turn to an effective description.

\section{Averaged evolution}

The study of the cosmological evolution of a fast oscillating scalar was first performed in [14]. Basically, if the oscillation frequency of the scalar field is much higher than the expansion rate of the universe, the cosmological evolution becomes independent of the periodic phase of the field at leading order. Consequently, the Einstein equations can be approximately solved averaging in time the energy-momentum tensor

$$
G^{\mu}{ }_{\nu}=8 \pi G\left\langle T^{\mu}{ }_{\nu}\right\rangle
$$

where

$$
\left\langle T^{\mu}{ }_{\nu}\right\rangle(t)=\frac{1}{T} \int_{t-T / 2}^{t+T / 2} T_{\nu}^{\mu}\left(t^{\prime}\right) \mathrm{d} t^{\prime} .
$$

If the field is periodic, we can consider an integer number of periods as the integration interval. However, similar results can be reached for fast-evolving bounded solutions averaging over time spans much bigger than the inverse of its frequency but much smaller than the inverse of the expansion rate, $\omega^{-1} \ll T \ll \mathcal{H}^{-1}$. The averaging error in both cases results $\mathcal{O}(\mathcal{H} T)$.

To leading order we can drop the averages of total time derivatives, so it can be proved [19] that

$$
\left\langle\dot{\phi}^{2} / a^{2}\right\rangle=-\left\langle\phi \ddot{\phi} / a^{2}\right\rangle=\left\langle V^{\prime}(\phi) \phi\right\rangle+\mathcal{O}\left(\frac{\mathcal{H}}{\omega_{\text {eff }}}\right),
$$

and with this result the effective equation of state can be written as

$$
w=\frac{\langle P\rangle}{\langle\rho\rangle}=\frac{\left\langle V^{\prime} \phi-2 V\right\rangle}{\left\langle V^{\prime} \phi+2 V\right\rangle}=\frac{n-2}{n+2}+\mathcal{O}\left(\frac{\mathcal{H}}{\omega_{\text {eff }}}\right)
$$


for power-law potentials $V(\phi) \propto \phi^{n}$. As it can be seen, a massive scalar field, $V=m^{2} \phi^{2} / 2$, would behave as CDM while a field with quartic self-interactions, $V=\lambda \phi^{4} / 4$, would behave as radiation.

The massive case is particularly simple and the equation of motion can be solved through a WKB expansion. Thanks to this adiabatic expansion in the parameter $\mathcal{O}(\mathcal{H} / m a)$ the averages can be performed explicitly, isolating the fast evolving factor and integrating by parts, as explained in [19].

In this work, we are interested in anharmonic corrections to the mass term (2.2). We can obtain the first order correction in $\lambda$ to the equation of state (3.4) assuming that the mass term is dominant, so the WKB expansion of [19] still holds to lowest order in $\lambda$

$$
\phi(\eta) \simeq \frac{\phi_{c}}{a^{3 / 2}} \cos \left(\int^{\eta} m a\left(\eta^{\prime}\right) \mathrm{d} \eta^{\prime}\right)+\mathcal{O}\left(\frac{\mathcal{H}}{m a}\right),
$$

With this WKB expression, we have, to lowest order in $\lambda$,

$$
\begin{aligned}
\left\langle\phi^{4}\right\rangle & \simeq \frac{3}{2}\left\langle\phi^{2}\right\rangle\left\langle\phi^{2}\right\rangle+\mathcal{O}\left(\frac{\mathcal{H}}{m a}\right), \\
\langle\rho\rangle & \simeq m^{2}\left\langle\phi^{2}\right\rangle+\mathcal{O}\left(\frac{\mathcal{H}}{m a}\right),
\end{aligned}
$$

so the first anharmonic correction to the equation of state is

$$
w \simeq \frac{3 \lambda}{8 m^{4}}\langle\rho\rangle \text {. }
$$

In this effective description the background evolution of the field is described through its density and its effective equation of state, using the conservation equation

$$
\dot{\rho}=-3 \mathcal{H}(1+w) \rho .
$$

where for the equation of state $w$ we will use the formula

$$
w=\frac{\frac{3 \lambda}{8 m^{4}} \rho}{1+\frac{9 \lambda}{8 m^{4}} \rho},
$$

that smoothly interpolates between the radiation-like $w \simeq 1 / 3$ and matter-like $w \simeq 0$ behaviour, whenever the quartic or quadratic part of the potential dominates. Now we can apply the same trick to the evolution of the perturbations. The equations of motion for the fluid variables are

$$
\begin{aligned}
\dot{\delta} & =3 \mathcal{H}\left(w-c_{s}^{2}\right) \delta-k u-\frac{1}{2}(1+w) \dot{h}, \\
\dot{u} & =-\mathcal{H}(1-3 w) u+k c_{s}^{2} \delta
\end{aligned}
$$

where $\delta \equiv\langle\delta \rho\rangle /\langle\rho\rangle, u \equiv(1+w)\langle v\rangle$ stand for averaged quantities and $w, c_{s}^{2}$ are the effective equation of state and sound speed. To complete the system there only remains to compute the effective sound speed

$$
c_{s}^{2}=\frac{\langle\delta P\rangle}{\langle\delta \rho\rangle} .
$$


In contrast with the adiabatic sound speed, the sound speed is gauge-dependent. But, as we will show now, the gauge ambiguities remain of order $\mathcal{O}\left(\mathcal{H} / \omega_{\text {eff }}\right)$ so our effective sound speed turns out to be gauge-independent. In fact, identical expressions have previously been obtained working in the comoving gauge [49] and in the Newtonian gauge [19]. To leading order we have

$$
\left\langle\partial_{\eta}(\dot{\phi} \delta \phi+\phi \delta \dot{\phi})\right\rangle=0+\mathcal{O}\left(\frac{\mathcal{H}}{\omega_{\text {eff }}}\right)
$$

Then, using (2.10) and (3.14) we can obtain the result

$$
\langle\dot{\phi} \dot{\delta \phi}\rangle=\frac{1}{2}\left\langle a^{2} V^{\prime} \delta \phi+\left(k^{2}+a^{2} V^{\prime \prime}\right) \phi \delta \phi\right\rangle+\Phi\left\langle a^{2} V^{\prime} \phi\right\rangle+\mathcal{O}\left(\frac{\mathcal{H}}{\omega_{\text {eff }}}\right),
$$

and finally compute the effective sound speed for a generic gauge

$$
\begin{aligned}
c_{s}^{2} & =\frac{\langle\delta P\rangle}{\langle\delta \rho\rangle}=\frac{\frac{1}{2}\left\langle V^{\prime} \delta \phi+\left((k / a)^{2}+V^{\prime \prime}\right) \phi \delta \phi-2 V^{\prime} \delta \phi\right\rangle-\Phi\left\langle\dot{\phi}^{2} / a^{2}-V^{\prime} \phi\right\rangle}{\frac{1}{2}\left\langle V^{\prime} \delta \phi+\left((k / a)^{2}+V^{\prime \prime}\right) \phi \delta \phi+2 V^{\prime} \delta \phi\right\rangle-\Phi\left\langle\dot{\phi}^{2} / a^{2}-V^{\prime} \phi\right\rangle}+\mathcal{O}\left(\frac{\mathcal{H}}{\omega_{\text {eff }}}\right) \\
& =\frac{\left\langle\left((k / a)^{2}+V^{\prime \prime}\right) \phi \delta \phi-V^{\prime} \delta \phi\right\rangle}{\left\langle\left((k / a)^{2}+V^{\prime \prime}\right) \phi \delta \phi+3 V^{\prime} \delta \phi\right\rangle}+\mathcal{O}\left(\frac{\mathcal{H}}{\omega_{\text {eff }}}\right)
\end{aligned}
$$

As we anticipated, the gauge ambiguities in the metric perturbations remain of order $\mathcal{O}\left(\mathcal{H} / \omega_{\text {eff }}\right)$, so the final expression holds in any gauge. Moreover, it can be rewritten in a manifestly gauge-invariant form substituting $\delta \phi$ by its gauge-invariant perturbation [59]

$$
\delta \phi^{(\mathrm{gi})}=\delta \phi+\dot{\phi}(B-\dot{E}),
$$

and using the relations

$$
\begin{aligned}
\left\langle V^{\prime} \dot{\phi}\right\rangle & =\left\langle\partial_{\eta}(V)\right\rangle=0+\mathcal{O}\left(\frac{\mathcal{H}}{\omega_{\text {eff }}}\right) \\
\left\langle V^{\prime \prime} \phi \dot{\phi}\right\rangle & =\left\langle\phi \partial_{\eta}\left(V^{\prime}\right)\right\rangle=-\left\langle V^{\prime} \dot{\phi}\right\rangle+\mathcal{O}\left(\frac{\mathcal{H}}{\omega_{\text {eff }}}\right)=0+\mathcal{O}\left(\frac{\mathcal{H}}{\omega_{\text {eff }}}\right)
\end{aligned}
$$

we obtain

$$
c_{s}^{2}=\frac{\left\langle\left((k / a)^{2}+V^{\prime \prime}\right) \phi \delta \phi^{(\mathrm{gi})}-V^{\prime} \delta \phi^{(\mathrm{gi})}\right\rangle}{\left\langle\left((k / a)^{2}+V^{\prime \prime}\right) \phi \delta \phi^{(\mathrm{gi})}+3 V^{\prime} \delta \phi^{(\mathrm{gi})}\right\rangle}+\mathcal{O}\left(\frac{\mathcal{H}}{\omega_{\mathrm{eff}}}\right) .
$$

This expression agrees with the result obtained in [19] working in the Newtonian gauge, so the same conclusions apply. In particular, a generic feature of this kind of models is a suppression of growth $c_{s}^{2} \simeq 1$ for small scales $k \gg \omega_{\text {eff. }}$ In the case of a power-law potential $V(\phi)=\frac{C}{n} \phi^{n}$, for large scales $k \ll \omega_{\text {eff }}$ we have

$$
c_{s}^{2}=\frac{n-2}{n+2}+\mathcal{O}\left(\frac{\mathcal{H}}{\omega_{\text {eff }}}\right)
$$


For a harmonic potential $n=2$, the zero-order term drops out and we must calculate the first-order corrections in $k$. Our potential of interest is a polynomial $V(\phi)=\frac{1}{2} m^{2} \phi^{2}+\frac{\lambda}{4} \phi^{4}$, a mass term plus an anharmonic correction, in this case we have [19]

$$
c_{s}^{2}=\frac{k^{2}}{4 m^{2} a^{2}}+\frac{3}{4} \frac{\lambda}{m^{4}} \rho,
$$

where $\rho$ is the energy density of the scalar field and the anharmonic correction is assumed to be small. In our numerical solution we will use an effective sound speed

$$
c_{s}^{2}=\frac{\left(\frac{k}{2 m a}\right)^{2}+\frac{3}{4} \frac{\lambda}{m^{4}} \rho}{1+\left(\frac{k}{2 m a}\right)^{2}+\frac{9}{4} \frac{\lambda}{m^{4}} \rho},
$$

suggested by the form of (3.17) and that smoothly interpolates between all the regimes of interest.

\section{Heuristic constraints on the non-harmonic contribution}

In this section we will discuss the simplest limits that constrain the model. With this objective let us assume a simple cosmology composed of radiation, cosmological constant and our scalar field

$$
\mathcal{H}^{2}=a^{2} H_{0}^{2}\left(\Omega_{\phi}(a)+\frac{\Omega_{\mathrm{rad}}}{a^{4}}+\Omega_{\Lambda}\right),
$$

where $\Omega_{i}=8 \pi G \rho_{i} /\left(3 H_{0}^{2}\right)$ are the abundances with $i \in\{\phi, \operatorname{rad}, \Lambda\}$ which correspond to scalar field, radiation and cosmological constant respectively.

- Limits on $\lambda$ from background evolution. The position of the peaks in the CMB temperature spectrum, especially the first one, is very sensitive to the amount of matter and the redshift of equality $z_{\text {eq }}$. We can assume that to have a viable model of dark matter this quantities remain essentially the same as in $\Lambda$ CDM. In this case, to have a dark matter behaviour that resemble CDM the anharmonic corrections at this time should be small

$$
1 \gg w \simeq \frac{3}{8} \frac{\lambda}{m^{4}} \rho_{\phi}\left(a_{\mathrm{eq}}\right) .
$$

This imposes an upper limit on $\lambda$, namely

$$
\lambda<\frac{8}{3} \frac{m^{4}}{\rho_{\phi}\left(a_{\mathrm{eq}}\right)},
$$

excluding the orange region in figure 1.

- Limits on $m$ from perturbation evolution. If $\lambda$ is small enough, the background evolution of the effective fluid is identical to $\Lambda$ CDM. In this case we can obtain limits from the behaviour of the perturbations. From (3.11) and (3.12) it can be 
seen that if we neglect the expansion rate, $c_{s}^{2} k^{2} \gg \mathcal{H}^{2}$, density perturbations evolve according to

$$
\ddot{\delta} \simeq-c_{s}^{2} k^{2} \delta .
$$

producing an oscillatory behaviour instead of the standard growth. To avoid a clear disagreement with observations, the effect of a non-negligible sound speed must be small

$$
c_{s}^{2} k^{2}<\mathcal{H}^{2}
$$

Translating into a lower bound in the allowed masses

$$
m>\frac{k^{2}}{2 a \mathcal{H}} .
$$

As before, we assume that $z_{\text {eq }}$ corresponds to the standard value and we apply the condition (4.6) at this redshift, that will give us the most conservative limit. For the wavenumber, we choose $k=0.2 \mathrm{Mpc}^{-1}$, the highest mode observed in LSS at the linear level. The constraint is

$$
m \gtrsim 10^{-26} \mathrm{eV}
$$

excluding the blue region in figure 1.

- Observable effects of anharmonic corrections. Finally, there is a region in the parameter space that we cannot yet exclude and where the effects of anharmonic corrections to the sound speed may be important

$$
c_{s}^{2} \simeq \frac{k^{2}}{4 m^{2} a^{2}}+\frac{3}{4} \frac{\lambda}{m^{4}} \rho_{\phi} .
$$

Imposing that the second term dominates over the harmonic contribution yields an upper bound on $\lambda$

$$
\lambda>\frac{k^{2} m^{2}}{3 \rho_{\phi} a^{2}},
$$

corresponding to a region that we cannot exclude right away, but where effects of the anharmonic corrections to the sound speed are to be expected.

An additional result that can be obtained from (4.5) is the Jeans wavenumber

$$
c_{s}^{2} k_{J}^{2}=\mathcal{H}^{2}
$$

Sub-Hubble modes below this Jeans wavenumber, $k<k_{J}$, grow while modes with $k>k_{J}$ are suppressed. In the massive case with $\lambda=0$ we obtain

$$
k_{J}^{2}=2 a \mathcal{H} m
$$

Now, since we have seen that the quartic correction affects the sound speed, it will also affect the Jeans scale. It is natural to ask what combination of parameters $(m, \lambda)$ can have a similar impact on structure formation as the case $(\tilde{m}, \tilde{\lambda}=0)$. To this end, we look for the combination that gives the same Jeans scale at the matter-radiation equality. Since 


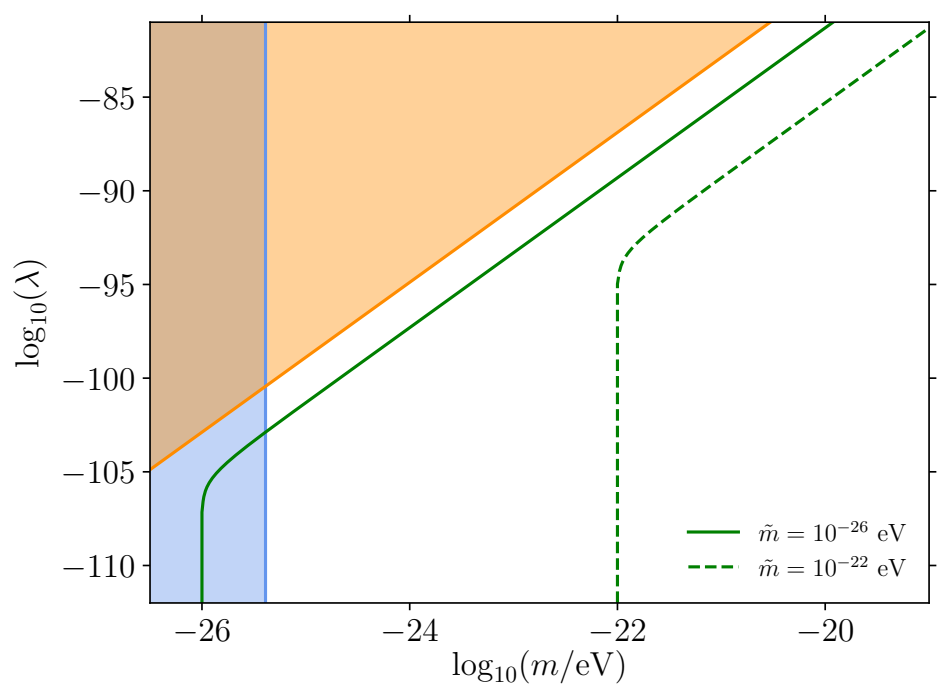

Figure 1. Different heuristic bounds. Orange region corresponds to the parameter-space excluded for the effects of $\lambda$ on the background evolution. In the blue region, the effect of a non-negligible sound speed results in a strong disagreement with observations, hence it is excluded. The green curves represent (4.12) for two different masses. According to the argument in the main text, points along each curve should give similar structure-formation results.

its scaling in time is not significantly modified, this simple estimate should capture the essential features of structure formation in both models. Equating both sound speeds and inserting the result (4.11) we have an estimate for $\lambda$

$$
\lambda=4.96 \times 10^{-100}\left(\frac{\tilde{m}}{10^{-24} \mathrm{eV}}\right)^{3}\left(\frac{1-r^{2}}{r^{4}}\right), \quad r \equiv \frac{\tilde{m}}{m} .
$$

This simple result suggests for instance that, at the linear level, structure formation should be similar in the models $\left(\tilde{m}=10^{-26} \mathrm{eV}, \tilde{\lambda}=0\right)$ and $\left(m=10^{-24} \mathrm{eV}, \lambda \simeq 4.96 \times 10^{-98}\right)$, a result that we will check with the full numerical solution. This estimate is represented in figure 1 for two different masses $\tilde{m}$.

After discussing some approximate bounds on our model and its physical origin, we will devote the next section to the full numerical solution.

\section{$5 \quad$ Numerical evolution and constraints}

We modify the publicly available Boltzmann code CLASS [54] and include this ultralight scalar field as a new species, that will assume the role of dark matter. Now, we summarize the key changes in the code and the evolution scheme chosen for the scalar field.

- At the background level, we start solving the equation (2.4) with initial conditions $\dot{\phi}=0$ and $\phi=\phi_{0}$. The initial value $\phi_{0}$ is chosen internally with a built-in shooting algorithm such as to match the energy density $\Omega_{\phi}$ (today) required. As a technical aside, it is critical to start with a sensible initial guess for $\phi_{0}$, so that the shooting algorithm converges quickly. In [18] the authors provide analytical formulae for the 
initial guess in the harmonic case, that works as well if the anharmonic corrections are small. If the quadratic and quartic terms are comparable it is more difficult to find analytical expressions that fit our purposes. In our case, we precompute an interpolation table for different values of $m, \lambda$ and $\phi_{0}$ yielding some value $\Omega_{\phi}\left(m, \lambda, \phi_{0}\right)$. We only compute a coarse table, so that we still use the shooting algorithm to adjust $\phi_{0}$ and achieve the desired precision in $\Omega_{\phi}$.

Another technical point involves the choice of $\dot{\phi}$ initial condition. Since CLASS starts the integration at a finite $a_{\text {ini }}=10^{-14}$, it is not strictly valid to set $\dot{\phi}\left(a_{\text {ini }}\right)=0$. The proper way to account for this finite initial time is to use the slow-roll approximation to obtain the analytic evolution at early times

$$
\dot{\phi} \simeq-\frac{a_{\mathrm{ini}}^{3} V\left(\phi_{0}\right)}{3 H_{0}} .
$$

However, we have checked that, in practice, evolution starts early enough to be equivalent to use $\dot{\phi}\left(a_{\text {ini }}\right)=0$. As long as the field starts in the slow-roll regime, the results are not significantly modified by the initial choice of $\dot{\phi}$.

With the initial conditions provided, the field starts its evolution frozen, slowly rolling down the potential until its natural frequency term in (2.4) dominates and it undergoes rapid oscillations. In this case it is computationally expensive to follow every oscillation so we turn to the averaged equations when $\sqrt{V^{\prime \prime}(\phi)}>3 H$.

In the averaged regime, we solve (3.9), matching continuously with the solution in the exact regime, and compute the pressure using the effective equation of state (3.10).

- At the perturbation level, we first solve (2.15) and (2.16) with adiabatic initial conditions $\delta=u=0$. For each mode $k$ we start the integration early enough to ensure that we start well within the exact regime, $\sqrt{V^{\prime \prime}(\phi)} \ll 3 H$. In the averaged regime, $\sqrt{V^{\prime \prime}(\phi)}>3 H$, we solve the equations (3.11) and (3.12) with the sound speed given by (3.24).

Some results for temperature and matter power spectra are shown in figures 2 and 3 . They show the impact of different choices of $m$ and $\lambda$, while the other cosmological parameters are fixed to their Planck [56] best-fit values. As anticipated, the main cosmological signature is the appearance of a cut-off in the matter power spectrum. This cut-off has already been discussed in the harmonic case [18]. In our case, we see that the anharmonic terms produce a similar effect.

\subsection{Physical effects}

The main physical effect responsible for the appearance of a cut-off in the matter power spectrum has already been discussed. In the averaged regime, the scalar field that supplies the dark matter component behaves like a fluid with a non-negligible sound speed. On small scales, above a certain Jeans scale $k_{J}$, the density perturbations oscillate and the growth is suppressed. This effect is illustrated in the figure 4 for modes above and below $k_{J}$. 

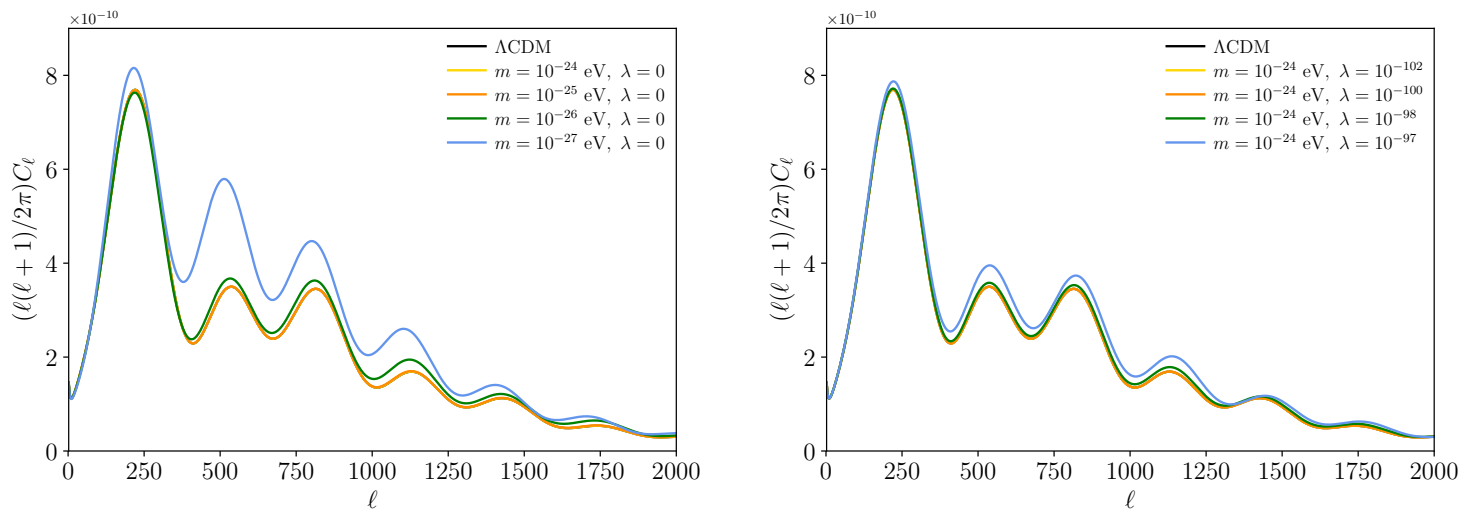

Figure 2. Temperature power spectrum. On the left, results for a massive scalar field without self-interaction. On the right, results for different self-interaction strengths for a mass that is indistinguishable from CDM with $\lambda=0$.
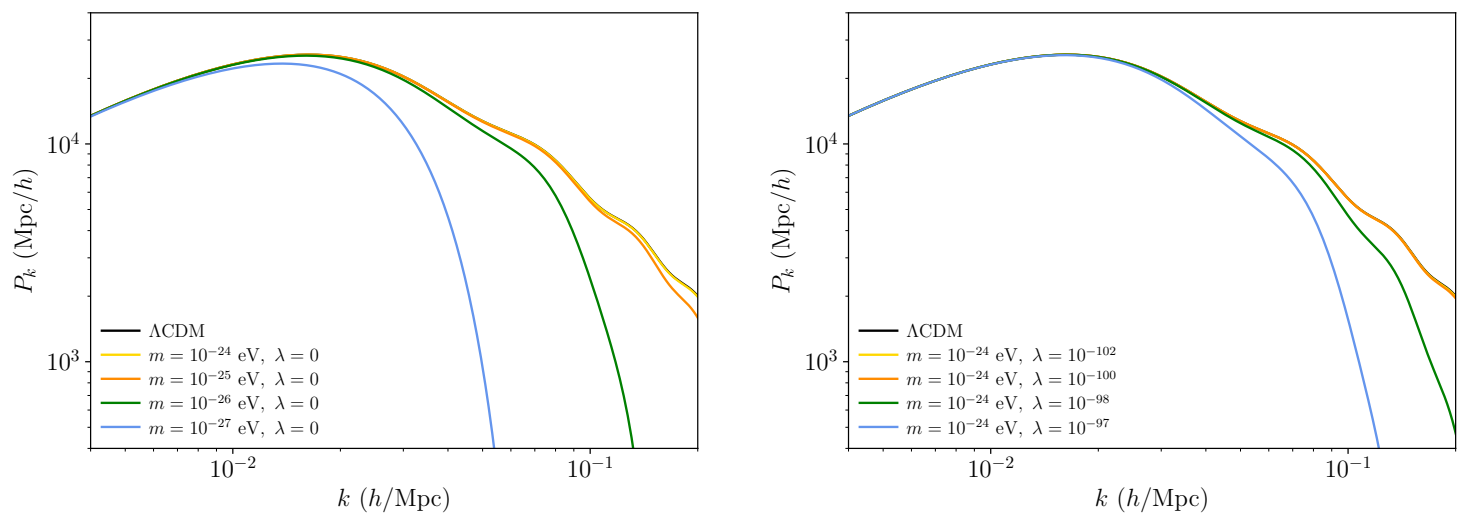

Figure 3. Matter power spectrum. On the left, results for a massive scalar field without selfinteraction. On the right, results for different self-interaction strengths for a mass that is indistinguishable from CDM with $\lambda=0$.
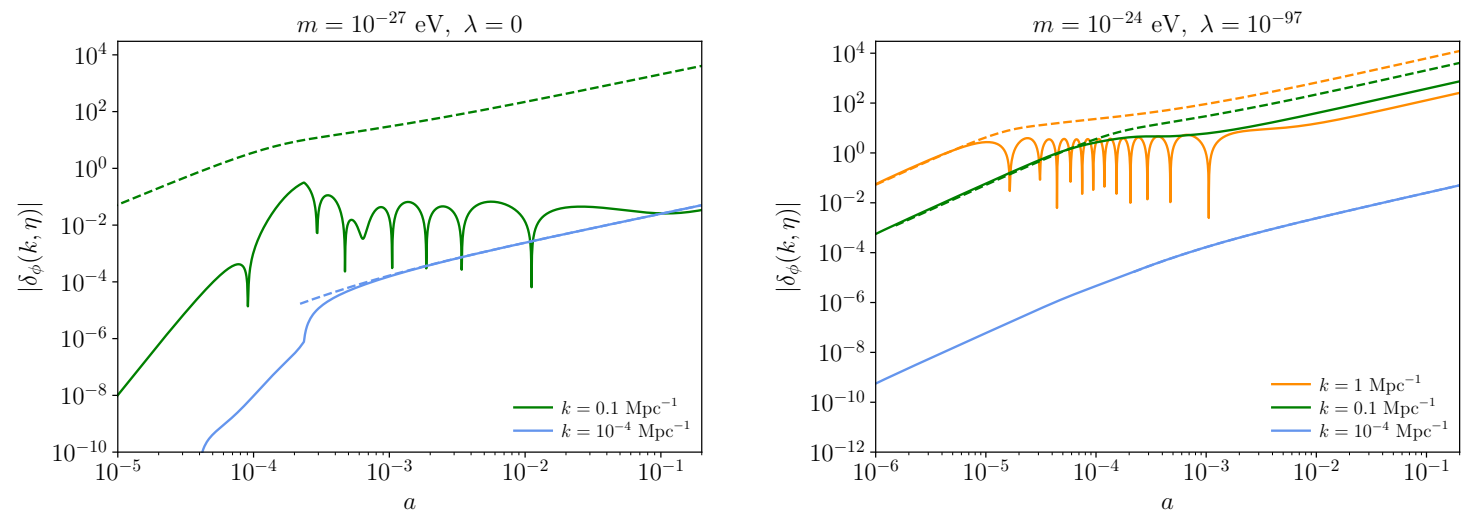

Figure 4. Evolution in time of the dark matter transfer functions compared to the standard $\Lambda \mathrm{CDM}$ evolution, represented by dotted lines, with Jeans scale at equality $k_{J}\left(z_{\mathrm{eq}}\right)=0.03 \mathrm{Mpc}^{-1}$ and $k_{J}\left(z_{\mathrm{eq}}\right)=0.11 \mathrm{Mpc}^{-1}$ respectively. 
In the case of the CMB temperature power spectrum, it is far more difficult to disentangle the physical effect responsible for each feature. We split the effects in two categories, those coming from the modified background evolution and those coming from the perturbations. Furthermore, we will refer to two extreme cases $\left(m=10^{-27}, \lambda=0\right)$ and ( $m=10^{-24}, \lambda=10^{-97}$ ) as $m$-case and $\lambda$-case respectively. To gain some insight into the CMB spectrum structure, we will rely on simplified, analytical estimates [60-62] and work in the Newtonian gauge. In particular, we will analyze the evolution of the background and the perturbations. The thermodynamic part of the evolution, i.e. redshift of recombination and decoupling, is not appreciably modified since it takes place well after equality, when the scalar field closely resembles CDM.

Background evolution. The modified equation of state (3.10) changes the background evolution, modifying in particular the redshift of matter-radiation equality $z_{\text {eq }}$ and in general the expansion history $a(\tau)$. In the $m$-case, the field transitions directly from the frozen value $w \simeq-1$ to a matter-like phase, while in the $\lambda$-case there is an intermediate radiation-like phase. There are two key effects

- First peak position. The position $\ell_{\text {peak }}$ of the first peak can be estimated as

$$
\theta_{\text {peak }}=\frac{\pi}{\ell_{\text {peak }}} \simeq \frac{\left.d_{s}\right|_{\text {dec }}}{\left.d_{a}\right|_{\text {dec }}}
$$

where the angular diameter $d_{a}$ distance is defined as

$$
\left.d_{a}\right|_{\mathrm{dec}}=a_{\mathrm{dec}} \int_{\eta_{\mathrm{dec}}}^{\eta_{0}} \mathrm{~d} \eta
$$

$\left.d_{s}\right|_{\text {dec }}$ is the sound horizon of the photon-baryon plasma evaluated at decoupling

$$
\left.d_{s}\right|_{\mathrm{dec}}=a_{\mathrm{dec}} \int_{0}^{\eta_{\mathrm{dec}}} c_{s \gamma} \mathrm{d} \eta
$$

and the sound speed for the baryon-photon plasma is

$$
c_{s \gamma}^{2}=\frac{1}{3(1+R)}, \quad R \equiv \frac{3 \rho_{b}}{4 \rho_{\gamma}} .
$$

The angular diameter distance is almost unaffected but the sound horizon is slightly modified. Compared to $\Lambda$ CDM we obtain relative deviations on $\ell_{\text {peak }}$ of about $+2 \%$, shift to the left, in the $m$-case and $-0.7 \%$, shift to the right, in the $\lambda$-case. Both are compatible with the tiny deviations observed in figure 2 .

- Damping envelope. Another physical scale that is modified is the diffusion length

$$
\left.\lambda_{D}\right|_{\mathrm{dec}}=a_{\mathrm{dec}}\left(\int_{0}^{\eta_{\mathrm{dec}}} \Gamma^{-1} \mathrm{~d} \eta\right)^{1 / 2},
$$

where

$$
\Gamma=a n_{e} \sigma_{T} x_{e}=-\dot{\kappa} x_{e}
$$


is the Thomson scattering rate. The diffusion length governs the damping envelope, $\mathrm{e}^{-\left(\ell / \ell_{D}\right)^{2}}$, through the relation

$$
\theta_{D}=\frac{\pi}{\ell_{D}} \simeq \frac{\left.\lambda_{D}\right|_{\mathrm{dec}}}{\left.d_{a}\right|_{\mathrm{dec}}}
$$

For a reference multipole $\ell=820$, corresponding to the third acoustic peak, in the $\lambda$-case we obtain a modified damping envelope that produces an enhancement of $6 \%$ compared to $\Lambda \mathrm{CDM}$, that can explain the overall increase of power in figure 2 . For the $m$-case, we obtain the puzzling result of a suppression of $0.7 \%$, in clear disagreement with the observed effect. However, we will shortly see how a novel effect in the perturbation evolution can account for this overall amplification.

Perturbation evolution. In the tightly coupled regime, the photon temperature fluctuation evolves according to

$$
\ddot{\Theta}_{0}+\frac{\dot{R}}{1+R} \dot{\Theta}_{0}+k^{2} c_{s \gamma}^{2} \Theta_{0}=-\frac{k^{2}}{3} \psi+\frac{\dot{R}}{1+R} \dot{\phi}+\ddot{\phi}
$$

In the standard scenario, ignoring slow changes in $R, \phi$ and $\psi$ from the expansion, we have

$$
\ddot{\Theta}_{0}+k^{2} c_{s \gamma}^{2} \Theta_{0} \simeq-\frac{k^{2}}{3} \psi \text {. }
$$

This produces an oscillatory pattern with frequency $\omega=k c_{s \gamma}$ and zero-point displaced by an amount $-(1+R) \psi$. The main part of the temperature Sachs-Wolfe effect comes from the contribution $\left.\left|\Theta_{0}+\psi\right|^{2}\right|_{\text {dec }}$, so the displacement of the zero-point of the oscillations gives the characteristic asymmetry between odd and even peaks in the CMB temperature spectrum. Our modification of dark matter produces two interrelated effects, oscillation and suppression of growth at small scales.

- Effects of suppression of growth at small scales. The suppression of dark matter density perturbations at small scales also suppresses the gravitational wells $\psi$, shifting the zero-point of the oscillation back to zero. This effect, alone, reduces the asymmetry among the peaks, decreasing the odd and increasing the even peaks. This explains the characteristic enhancement of the second peak with respect to the third one in figure 2.

- Effects of oscillatory behaviour. There only remains to explain one effect, the striking gain in peak amplitude in the $m$-case. According to the modification in the damping envelope, the peaks should be slightly suppressed and their enhancement is actually related to a resonance effect. In the standard scenario, the term $\psi$ behaves like a constant external force, shifting the equilibrium position of the photon oscillations. In our case, it is not constant anymore, but oscillates with a frequency $k c_{s}$ given by the sound speed of the dark matter perturbations (3.24). These two frequencies, $k c_{s}$ and $k c_{s \gamma}$, are comparable for a range of $k$ values, as shown in figure 5 , producing a resonant effect that increases the height of the peaks, as shown in figure 6 . 


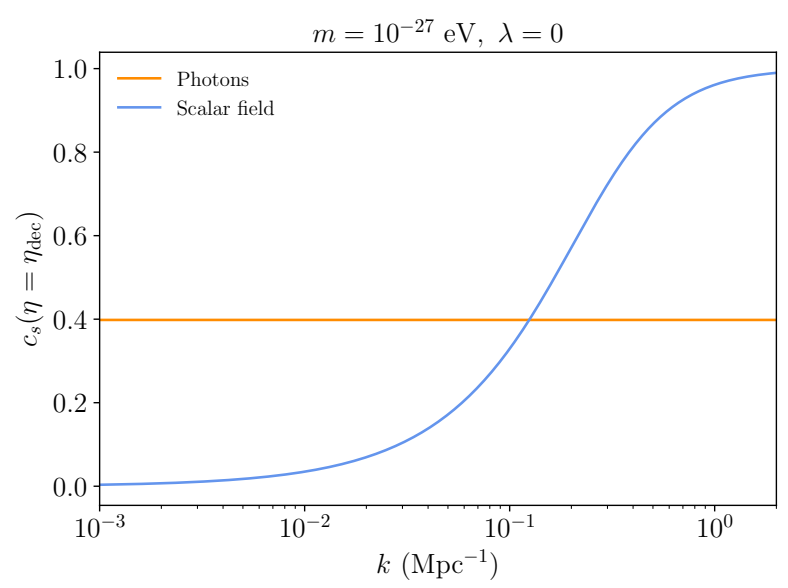

Figure 5. Sound speed at decoupling for photons and dark matter. Around $k \simeq 0.1 \mathrm{Mpc}^{-1}$ the sound speed for both fluids, hence the oscillation frequency too, are close and we have a resonant driving.

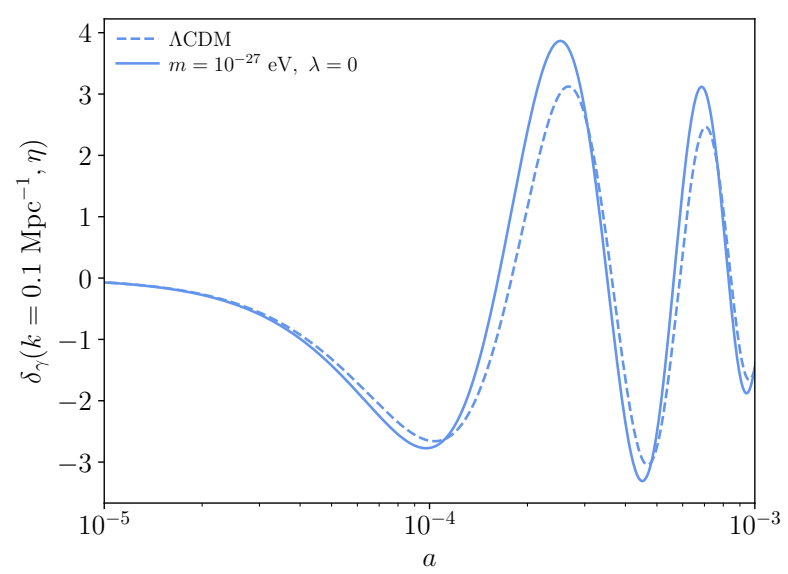

Figure 6. Evolution in time of the mode $k=0.1 \mathrm{Mpc}^{-1}$, corresponding approximately to the fifth acoustic peak, until decoupling.

Moreover, since according to (3.24) the scale of the crossover in figure 5 evolves $\propto a$, as we go from decoupling back in time it moves to smaller $k$. That is to say, although the crossover at decoupling is located around $k \simeq 0.1 \mathrm{Mpc}^{-1}$, smaller $k$ have also fulfilled the resonance condition at previous times, so they have also got amplified.

\subsection{Observational constraints}

To compare this model with CMB and LSS observations and refine the heuristic constraints obtained in section 4, we use the public parameter-extraction code MontePython [55]. We will compare our results with two different data sets: CMB measurements by Planck and large-scale structure information by WiggleZ [57]. We perform two analysis, Planck only and Planck+WiggleZ. In each case we vary the six $\Lambda$ CDM base model parameters, in addition to the foreground parameters, plus $m$ and $\lambda$, the mass and anharmonic parameter. We choose logarithmic priors in our model parameters, as shown in table 1. 


\begin{tabular}{|lcc|}
\hline Parameter & minimum & maximum \\
\hline$\Omega_{b} h^{2}$ & - & - \\
$\Omega_{\phi} h^{2}$ & - & - \\
$h$ & - & - \\
$\log \left(10^{10} A_{s}\right)$ & - & - \\
$n_{s}$ & - & - \\
$\tau_{\text {reio }}^{2}$ & 0.04 & - \\
$\log _{10}(m / \mathrm{eV})$ & -26 & -23.3 \\
$\log _{10}(\lambda)$ & -111 & -98 \\
\hline
\end{tabular}

Table 1. Prior ranges on the base $\Lambda \mathrm{CDM}$ parameters and the model parameters $m$ and $\lambda$. A symbol - means that there is no prior. Additionally, the fixed parameters include the neutrino properties. In our case, two massless neutrinos plus a massive one with $m=0.06 \mathrm{eV}$, such that $N_{\text {eff }}=3.046$ and $m_{\nu} / \Omega_{\nu}=93.14 \mathrm{eV}$.

It is important to note that to perform an accurate comparison with LSS data we must restrict our analysis to linear scales $k \lesssim 0.2 h / \mathrm{Mpc}$. The non-linear module in CLASS includes HALOFit [63], but since it has not been calibrated for our model we restrict our analysis to linear scales without non-linear corrections. It is to be expected that, in the future, as more $N$-body simulations with ultralight fields become available, non-linear information will allow us to tighten the constraints.

We do not observe any significant degeneracy between $m, \lambda$ and the rest of cosmological parameters. Best-fit results are shown in table 2, while the marginalized countour for our model parameters is represented in figure 7 .

\section{Conclusions}

The presence of self-interactions in the ultralight field potential can lead to the appearance of new background-evolution phases, like the radiation-like due to our quartic potential. This modified background evolution, and especially its critical effect on the sound speed of dark matter perturbations, can lead to significant differences from observations. The observational signatures of the anharmonic contribution are similar to the mass term, the most prominent being the appearance of a cut-off in the matter power spectrum. This produces constraints for masses that would be otherwise indistinguishable from CDM, i.e. $m \gtrsim 10^{-24} \mathrm{eV}$. Our constraints on $\lambda$ complement other bounds present in the literature, e.g. [51]. This bounds on $\lambda$ follow a scaling law with $m^{4}$ according to (4.3). We can extrapolate the results to higher masses using the $2 \sigma$ region of figure 7 , obtaining an approximate constraint on $\lambda$

$$
\log _{10}(\lambda)<-91.86+4 \log _{10}\left(\frac{m}{10^{-22} \mathrm{eV}}\right),
$$

for masses $m>10^{-24} \mathrm{eV}$.

So far, we have only analyzed linear observables, but in fact larger effects on nonlinear scales are expected. The available parameter space could be further constrained in 


\begin{tabular}{|lcc|}
\hline Base parameters & Planck & Planck+WiggleZ \\
\hline$\Omega_{b} h^{2}$ & $0.02223 \pm 0.00047$ & $0.02212_{-0.00041}^{+0.00042}$ \\
$\Omega_{\phi} h^{2}$ & $0.1189_{-0.0041}^{+0.0044}$ & $0.1204_{-0.0034}^{+0.0032}$ \\
$h$ & $0.677 \pm 0.019$ & $0.670_{-0.014}^{+0.016}$ \\
$\log \left(10^{10} A_{s}\right)$ & $3.070_{-0.053}^{+0.056}$ & $3.057_{-0.041}^{+0.046}$ \\
$n_{s}$ & $0.965_{-0.021}^{+0.016}$ & $0.963_{-0.010}^{+0.011}$ \\
$\tau_{\text {reio }}^{2}$ & $0.070_{-0.029}^{+0.028}$ & $0.061_{-0.021}^{+0.024}$ \\
$\log _{10}(m / \mathrm{eV})$ & $>-24.5$ & $>-24.4$ \\
$\log _{10}(\lambda)$ & - & $<-99.0$ \\
\hline Derived parameters & & \\
\hline$z_{\text {reio }}$ & $9.2_{-2.7}^{+2.6}$ & $8.4_{-2.1}^{+2.2}$ \\
$\Omega_{\Lambda}$ & $0.690_{-0.027}^{+0.024}$ & $0.681 \pm 0.021$ \\
$Y_{\mathrm{He}}$ & $0.24778 \pm 0.00020$ & $0.24773 \pm 0.00018$ \\
$100 \theta_{s}$ & $1.04193_{-0.00099}^{+0.00098}$ & $1.04182_{-0.00083}^{+0.00084}$ \\
\hline
\end{tabular}

Table 2. Best fit results with $95 \%$ confidence level.

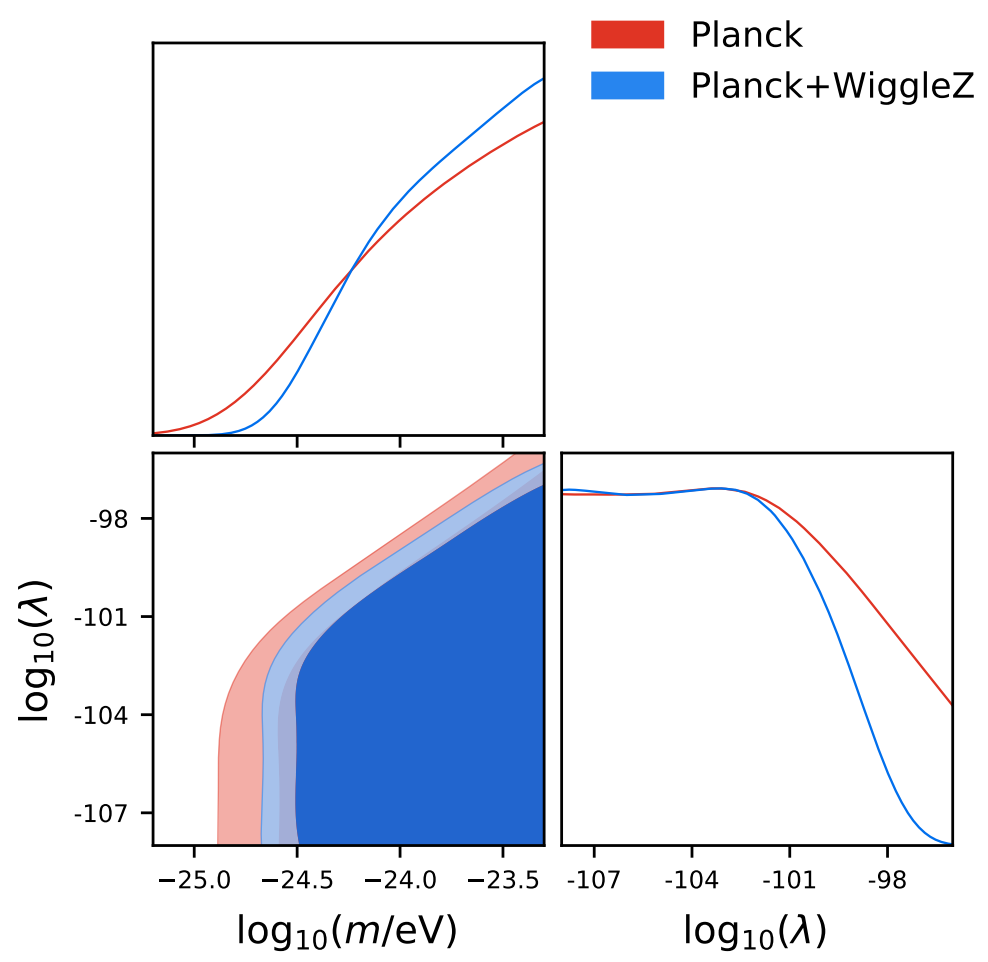

Figure 7. Contour plots with $95 \%$ and $99 \%$ confidence levels and 1d marginalized distributions. 
the future using cosmological information with non-linear observables, as more simulations with ultralight fields become available. Even without non-linear information, using the formula (4.12) one could put forward the proposal that similar results on structure formation could be obtained for higher masses with a positive $\lambda$. For instance, results for $\tilde{m}=10^{-22} \mathrm{eV}$ might be reproduced with masses $m \simeq 10^{-5} \mathrm{eV}$ adding a self-interaction of the order of $\lambda \simeq 10^{-24}$, very close to the limit that can be obtained from (6.1). Nevertheless, a definitive answer to this suggestive proposal require a fully non-linear analysis.

\section{Acknowledgments}

this work has been supported by the MINECO (Spain) projects FIS2014-52837-P, FIS201678859-P(AEI/FEDER, UE), and Consolider-Ingenio MULTIDARK CSD2009-00064.

Open Access. This article is distributed under the terms of the Creative Commons Attribution License (CC-BY 4.0), which permits any use, distribution and reproduction in any medium, provided the original author(s) and source are credited.

\section{References}

[1] P.W. Graham et al., Experimental searches for the axion and axion-like particles, Ann. Rev. Nucl. Part. Sci. 65 (2015) 485 [arXiv:1602.00039] [InSPIRE].

[2] H. Goldberg, Constraint on the photino mass from cosmology, Phys. Rev. Lett. 50 (1983) 1419 [Erratum ibid. 103 (2009) 099905] [INSPIRE].

[3] J.R. Ellis et al., Supersymmetric relics from the Big Bang, Nucl. Phys. B 238 (1984) 453 [INSPIRE].

[4] B. Carr, F. Kuhnel and M. Sandstad, Primordial black holes as dark matter, Phys. Rev. D 94 (2016) 083504 [arXiv: 1607.06077] [INSPIRE].

[5] J.P. Ostriker and P.J. Steinhardt, New light on dark matter, Science 300 (2003) 1909 [astro-ph/0306402] [INSPIRE].

[6] D.H. Weinberg et al., Cold dark matter: controversies on small scales, Proc. Nat. Acad. Sci. 112 (2015) 12249 [arXiv:1306.0913] [InSPIRE].

[7] A. Pontzen and F. Governato, Cold dark matter heats up, Nature 506 (2014) 171 [arXiv: 1402.1764] [INSPIRE].

[8] M. Boylan-Kolchin, J.S. Bullock and M. Kaplinghat, Too big to fail? The puzzling darkness of massive Milky Way subhaloes, Mon. Not. Roy. Astron. Soc. 415 (2011) L40 [arXiv: 1103.0007] [INSPIRE].

[9] B. Moore et al., Dark matter substructure within galactic halos, Astrophys. J. 524 (1999) L19 [astro-ph/9907411] [INSPIRE].

[10] W.J.G. de Blok, The core-cusp problem, Adv. Astron. 2010 (2010) 789293 [arXiv: 0910.3538] [INSPIRE].

[11] J. Oñorbe et al., Forged in FIRE: cusps, cores and baryons in low-mass dwarf galaxies, Mon. Not. Roy. Astron. Soc. 454 (2015) 2092 [arXiv: 1502.02036] [INSPIRE]. 
[12] W. Hu, R. Barkana and A. Gruzinov, Cold and fuzzy dark matter, Phys. Rev. Lett. 85 (2000) 1158 [astro-ph/0003365] [INSPIRE].

[13] H.-Y. Schive, T. Chiueh and T. Broadhurst, Cosmic structure as the quantum interference of a coherent dark wave, Nature Phys. 10 (2014) 496 [arXiv:1406.6586] [INSPIRE].

[14] M.S. Turner, Coherent scalar field oscillations in an expanding universe, Phys. Rev. D 28 (1983) 1243 [INSPIRE].

[15] M.C. Johnson and M. Kamionkowski, Dynamical and gravitational instability of oscillating-field dark energy and dark matter, Phys. Rev. D 78 (2008) 063010 [arXiv: 0805.1748] [INSPIRE].

[16] J.-c. Hwang and H. Noh, Axion as a Cold Dark Matter candidate, Phys. Lett. B 680 (2009) 1 [arXiv:0902.4738] [INSPIRE].

[17] C.-G. Park, J.-c. Hwang and H. Noh, Axion as a cold dark matter candidate: low-mass case, Phys. Rev. D 86 (2012) 083535 [arXiv:1207.3124] [InSPIRE].

[18] R. Hlozek et al., A search for ultralight axions using precision cosmological data, Phys. Rev. D 91 (2015) 103512 [arXiv:1410.2896] [INSPIRE].

[19] J.A.R. Cembranos, A.L. Maroto and S.J. Núñez Jareño, Cosmological perturbations in coherent oscillating scalar field models, JHEP 03 (2016) 013 [arXiv:1509.08819] [INSPIRE].

[20] R. Hložek et al., Future CMB tests of dark matter: ultralight axions and massive neutrinos, Phys. Rev. D 95 (2017) 123511 [arXiv: 1607.08208] [INSPIRE].

[21] R. Hlozek, D.J.E. Marsh and D. Grin, Using the full power of the cosmic microwave background to probe axion dark matter, Mon. Not. Roy. Astron. Soc. 476 (2018) 3063 [arXiv: 1708.05681] [INSPIRE].

[22] J.A.R. Cembranos et al., Isotropy theorem for cosmological vector fields, Phys. Rev. D 86 (2012) 021301 [arXiv: 1203.6221] [INSPIRE].

[23] J.A.R. Cembranos, A.L. Maroto and S.J. Núñez Jareño, Perturbations of ultralight vector field dark matter, JHEP 02 (2017) 064 [arXiv:1611.03793] [INSPIRE].

[24] J.A.R. Cembranos, A.L. Maroto and S.J. Núñez Jareño, Isotropy theorem for cosmological Yang-Mills theories, Phys. Rev. D 87 (2013) 043523 [arXiv:1212.3201] [InSPIRE].

[25] J.A.R. Cembranos, A.L. Maroto and S.J. Núñez Jareño, Isotropy theorem for arbitrary-spin cosmological fields, JCAP 03 (2014) 042 [arXiv:1311.1402] [INSPIRE].

[26] R.D. Peccei and H.R. Quinn, CP conservation in the presence of instantons, Phys. Rev. Lett. 38 (1977) 1440 [INSPIRE].

[27] F. Wilczek, Problem of strong $p$ and $t$ invariance in the presence of instantons, Phys. Rev. Lett. 40 (1978) 279 [INSPIRE].

[28] S. Weinberg, A new light boson?, Phys. Rev. Lett. 40 (1978) 223 [INSPIRE].

[29] D.J.E. Marsh, Axion cosmology, Phys. Rept. 643 (2016) 1 [arXiv:1510.07633] [INSPIRE].

[30] L. Hui, J.P. Ostriker, S. Tremaine and E. Witten, Ultralight scalars as cosmological dark matter, Phys. Rev. D 95 (2017) 043541 [arXiv:1610.08297] [INSPIRE].

[31] A. Sarkar et al., The effects of the small-scale DM power on the cosmological neutral hydrogen (HI) distribution at high redshifts, JCAP 04 (2016) 012 [arXiv: 1512.03325] [INSPIRE]. 
[32] T. Kobayashi et al., Lyman- $\alpha$ constraints on ultralight scalar dark matter: implications for the early and late universe, Phys. Rev. D 96 (2017) 123514 [arXiv:1708.00015] [InSPIRE].

[33] C. Abel et al., Search for axionlike dark matter through nuclear spin precession in electric and magnetic fields, Phys. Rev. X 7 (2017) 041034 [arXiv:1708.06367] [INSPIRE].

[34] Y.V. Stadnik and V.V. Flambaum, Can dark matter induce cosmological evolution of the fundamental constants of Nature?, Phys. Rev. Lett. 115 (2015) 201301 [arXiv:1503.08540] [INSPIRE].

[35] Y.V. Stadnik and V.V. Flambaum, Improved limits on interactions of low-mass spin-0 dark matter from atomic clock spectroscopy, Phys. Rev. A 94 (2016) 022111 [arXiv: 1605.04028] [INSPIRE].

[36] N. Banik, A.J. Christopherson, P. Sikivie and E.M. Todarello, New astrophysical bounds on ultralight axionlike particles, Phys. Rev. D 95 (2017) 043542 [arXiv:1701.04573] [INSPIRE].

[37] S. Hirano, J.M. Sullivan and V. Bromm, First star formation in ultralight particle dark matter cosmology, Mon. Not. Roy. Astron. Soc. 473 (2018) L6 [arXiv:1706. 00435] [INSPIRE].

[38] J.P. Conlon et al., Projected bounds on ALPs from Athena, Mon. Not. Roy. Astron. Soc. 473 (2018) 4932 [arXiv:1707.00176] [INSPIRE].

[39] R. Brito et al., Stochastic and resolvable gravitational waves from ultralight bosons, Phys. Rev. Lett. 119 (2017) 131101 [arXiv:1706.05097] [INSPIRE].

[40] R. Brito et al., Gravitational wave searches for ultralight bosons with LIGO and LISA, Phys. Rev. D 96 (2017) 064050 [arXiv: 1706.06311] [INSPIRE].

[41] A. Sarkar, S.K. Sethi and S. Das, The effects of the small-scale behaviour of dark matter power spectrum on CMB spectral distortion, JCAP 07 (2017) 012 [arXiv:1701.07273] [INSPIRE].

[42] J.A.D. Diacoumis and Y.Y.Y. Wong, Using CMB spectral distortions to distinguish between dark matter solutions to the small-scale crisis, JCAP 09 (2017) 011 [arXiv:1707.07050] [INSPIRE].

[43] L.A. Ureña-López and A.X. Gonzalez-Morales, Towards accurate cosmological predictions for rapidly oscillating scalar fields as dark matter, JCAP 07 (2016) 048 [arXiv:1511.08195] [INSPIRE].

[44] F.X.L. Cedeño, A.X. González-Morales and L.A. Ureña-López, Cosmological signatures of ultralight dark matter with an axionlike potential, Phys. Rev. D 96 (2017) 061301 [arXiv:1703.10180] [INSPIRE].

[45] V. Desjacques, A. Kehagias and A. Riotto, Impact of ultralight axion self-interactions on the large scale structure of the Universe, Phys. Rev. D 97 (2018) 023529 [arXiv:1709.07946] [INSPIRE].

[46] J. Fan, Ultralight Repulsive Dark Matter and BEC, Phys. Dark Univ. 14 (2016) 84 [arXiv: 1603.06580] [INSPIRE].

[47] M. Khlopov, B.A. Malomed and I.B. Zeldovich, Gravitational instability of scalar fields and formation of primordial black holes, Mon. Not. Roy. Astron. Soc. 215 (1985) 575 [INSPIRE].

[48] J. Goodman, Repulsive dark matter, New Astron. 5 (2000) 103 [astro-ph/0003018] [INSPIRE]. 
[49] B. Li, T. Rindler-Daller and P.R. Shapiro, Cosmological constraints on Bose-Einstein-Condensed scalar field dark matter, Phys. Rev. D 89 (2014) 083536 [arXiv: 1310.6061] [INSPIRE].

[50] A. Suárez and P.-H. Chavanis, Cosmological evolution of a complex scalar field with repulsive or attractive self-interaction, Phys. Rev. D 95 (2017) 063515 [arXiv:1608.08624] [INSPIRE].

[51] P.S.B. Dev, M. Lindner and S. Ohmer, Gravitational waves as a new probe of Bose-Einstein condensate Dark Matter, Phys. Lett. B 773 (2017) 219 [arXiv:1609.03939] [InSPIRE].

[52] B. Li, P.R. Shapiro and T. Rindler-Daller, Bose-Einstein-condensed scalar field dark matter and the gravitational wave background from inflation: new cosmological constraints and its detectability by LIGO, Phys. Rev. D 96 (2017) 063505 [arXiv:1611.07961] [InSPIRE].

[53] T. Rindler-Daller and P.R. Shapiro, Angular momentum and vortex formation in Bose-Einstein-condensed cold dark matter haloes, Mon. Not. Roy. Astron. Soc. 422 (2012) 135 [arXiv: 1106.1256] [INSPIRE].

[54] D. Blas, J. Lesgourgues and T. Tram, The Cosmic Linear Anisotropy Solving System (CLASS) II: approximation schemes, JCAP 07 (2011) 034 [arXiv:1104.2933] [INSPIRE].

[55] B. Audren, J. Lesgourgues, K. Benabed and S. Prunet, Conservative constraints on early cosmology: an illustration of the Monte Python cosmological parameter inference code, JCAP 02 (2013) 001 [arXiv: 1210.7183] [INSPIRE].

[56] Planck collaboration, P.A.R. Ade et al., Planck 2015 results. XIII. Cosmological parameters, Astron. Astrophys. 594 (2016) A13 [arXiv:1502.01589] [INSPIRE].

[57] D. Parkinson et al., The WiggleZ Dark Energy Survey: Final data release and cosmological results, Phys. Rev. D 86 (2012) 103518 [arXiv:1210.2130] [InSPIRE].

[58] A. Diez-Tejedor and D.J.E. Marsh, Cosmological production of ultralight dark matter axions, arXiv: 1702.02116 [INSPIRE].

[59] V.F. Mukhanov, H.A. Feldman and R.H. Brandenberger, Theory of cosmological perturbations. Part 1. Classical perturbations. Part 2. Quantum theory of perturbations. Part 3. Extensions, Phys. Rept. 215 (1992) 203 [InSPIRE].

[60] W. Hu and N. Sugiyama, Small scale cosmological perturbations: an analytic approach, Astrophys. J. 471 (1996) 542 [astro-ph/9510117] [INSPIRE].

[61] S. Weinberg, Cosmology, Oxford University Press, Oxford U.K. (2008).

[62] J. Lesgourgues, Cosmological Perturbations, in the proceedings of the Theoretical Advanced Study Institute in Elementary Particle Physics: Searching for New Physics at Small and Large Scales (TASI 2012), June 4-29, Boulder, Colorado, U.S.A. (2012), arXiv:1302.4640 [INSPIRE].

[63] S. Bird, M. Viel and M.G. Haehnelt, Massive neutrinos and the non-linear matter power spectrum, Mon. Not. Roy. Astron. Soc. 420 (2012) 2551 [arXiv:1109.4416] [inSPIRE]. 